\title{
ZERO-DIVISOR GRAPHS OF $2 \times 2$ UPPER TRIANGULAR MATRIX RINGS OVER $\mathbb{Z}_{n}$
}

\author{
TODD FENSTERMACHER AND ETHAN GEGNER
}

\begin{abstract}
Istvan Beck introduced the zero-divisor graph in 1988. We explore the directed and undirected zero-divisor graphs of the rings of $2 \times 2$ upper triangular matrices $\bmod n$, denoted by $\Gamma\left(T_{2}(n)\right)$ and $\tilde{\Gamma}\left(T_{2}(n)\right)$, respectively. For prime $p$, we completely characterize the graph $\Gamma\left(T_{2}(p)\right)$ by partitioning $T_{2}(p)$, and prove several key properties of the graphs using this approach. We establish additional properties of $\Gamma\left(T_{2}(n)\right)$ for arbitrary $n$. We prove that $\Gamma\left(T_{2}(n)\right)$ is Hamiltonian if and only if $n$ is prime, and we give explicit formulas for the edge connectivity and clique number of $\Gamma\left(T_{2}(n)\right)$ in terms of the prime factorization of $n$.
\end{abstract}

\section{INTRODUCTION}

The zero-divisor graph of a ring $R$ is a graph whose vertices are the nonzero zero-divisors of $R$. The zero-divisor graph was introduced by Istvan Beck in his work [5], where he employed the concept to solve graph coloring problems. Anderson and Naseer expanded upon Beck's work, and provided a counterexample to his conjecture that the chromatic and clique numbers are equal for the zero-divisor graph of any commutative ring [1]. Redmond introduced multiple versions of zero-divisor graphs and extended the definition to non-commutative rings in [12]. Much of our work is motivated and aided by Redmond's research [12].

Since Beck's work, the motivation for studying zero-divisor graphs has shifted from graph colorings to understanding the ring zero-divisors themselves. When present, zero-divisors are important elements of a ring. Zerodivisor graphs enable us to study the structure of their respective rings using graph theory, as opposed to more conventional methods.

Here we study the zero-divisor graphs of the rings $T_{2}(n)$ under matrix addition and multiplication, where $T_{2}(n)$ denotes the set of upper-triangular matrices with elements in $\mathbb{Z}_{n}$. A large amount of research has been devoted to the study of the zero-divisor graphs of commutative rings, see [1] and [13]. However, research in the area of non-commutative rings is less copious, although some authors have established general results, such as [6] and 


\section{T. FENSTERMACHER AND E. GEGNER}

[12]. In addition to their applications in graph coloring, zero-divisor graphs provide a link between graph and number theory.

We study the relationship between the ring- and graph-theoretic properties of the ring $T_{2}(n)$ and the directed and undirected zero-divisor graphs of $T_{2}(n)$. The directed and undirected graphs of $T_{2}(n)$ are denoted by $\Gamma\left(T_{2}(n)\right)$ and $\tilde{\Gamma}\left(T_{2}(n)\right)$, respectively. We give Behboodi and Beyranvand's result [6] that $\tilde{\Gamma}(R)$ is connected for every ring $R$, and we show that $\Gamma\left(T_{2}(n)\right)$ is connected using a result due to Redmond [13]. If $n$ is prime, then the chromatic number of $\Gamma\left(T_{2}(n)\right)$ is $n+1$. If $p$ is the smallest prime factor of $n$, then the edge connectivity of $\Gamma\left(T_{2}(n)\right)$ is $p-1$ and the edge connectivity of $\tilde{\Gamma}(R)$ is $p^{2}-1$. Finally, we prove that $\Gamma\left(T_{2}(n)\right)$ is Hamiltonian if and only if $n$ is prime and vertex-pancyclic if and only if $n$ is an odd prime, and we establish an explicit formula for the clique number of $\Gamma\left(T_{2}(n)\right)$.

\section{Preliminaries}

Let $G$ be a graph, and let $a, b$ be vertices of $G$. If $G$ is a directed graph, then we write $a \rightarrow b$ if there is an edge of $G$ from $a$ to $b$. If both $a \rightarrow b$ and $b \rightarrow a$, then we say there is a multiple edge between $a$ and $b$. If $G$ is undirected, then we write $a-b$ if there is an edge of $G$ from $a$ to $b$. Throughout the paper, $\mathbf{0}$ denotes the $2 \times 2$ zero matrix. If $R$ is a commutative ring, and $a$ is an element of $R$, then $\langle a\rangle$ denotes the ideal of $R$ generated by $a$.

Most authors define only undirected complete graphs. However, for our purposes it will be useful to provide a definition that may be applied to directed complete graphs as well. Thus, we call a directed graph $G$ with $n$ vertices a complete graph on $n$ vertices if for all vertices $x, y$ of $G$ with $x \neq y$ there is a multiple edge between $x$ and $y$. We denote such a complete graph by $K^{n}$.

Throughout the following definitions, $R$ denotes an arbitrary ring.

Definition 2.1. An element $a \in R$ is a zero-divisor of $R$ if $a \neq 0$ and there exists some $b \in R$ such that $a b=0$ or $b a=0$ in $R$. If $a b=0(b a=0)$, then we say that $a$ is a left (right) zero-divisor of $R$. The set of all zero-divisors of $R$ is denoted by $Z(R)$. The set of all left (right) zero-divisors of $R$ is denoted by $Z^{L}(R)\left(Z^{R}(R)\right)$.

We denote the set of all upper-triangular $2 \times 2$ matrices with elements in $\mathbb{Z}_{n}$ by $T_{2}(n)$. Also, in order to simplify notation, we denote the set of all zero-divisors of $T_{2}(n)$ by $Z_{2}(n)$ instead of $Z\left(T_{2}(n)\right)$.

Definition 2.2. $\Gamma(R)$ is the directed zero-divisor graph of $R$ where $a \rightarrow b$ if $a, b \in Z(R)$ and $a b=0$. 


\section{ZERO-DIVISOR GRAPHS OF $2 \times 2$ MATRIX RINGS OVER $\mathbb{Z}_{n}$}

Definition 2.3. $\tilde{\Gamma}(R)$ is the undirected zero-divisor graph of $R$ where $a-b$ if $a, b \in Z(R)$ and $a b=0$ or $b a=0$.

Definition 2.4. $\bar{\Gamma}(R)$ is the strict zero-divisor graph of $R$ where $a-b$ if $a, b \in Z(R)$ and $a b=\mathbf{0}$ and $b a=\mathbf{0}$.

Note that the strict graph of $R$ is a subgraph of the undirected graph.

\section{REsults}

Lemma 3.1. Let $n \geq 2$, and let $A=\left(\begin{array}{cc}a & b \\ 0 & c\end{array}\right) \in T_{2}(n)$ with $A \neq \mathbf{0}$. Then $A \in Z_{2}(n)$ if and only if $\operatorname{gcd}(a, n) \neq 1$ or $\operatorname{gcd}(c, n) \neq 1$.

Proof. Assume $\operatorname{gcd}(a, n)=\operatorname{gcd}(c, n)=1$. Since $A \in Z_{2}(n)$, there must exist some $X=\left(\begin{array}{cc}x & y \\ 0 & z\end{array}\right) \in Z_{2}(n)$ such that

$$
A X=\left(\begin{array}{cc}
a x & a y+b z \\
0 & c z
\end{array}\right)=\mathbf{0}
$$

or

$$
X A=\left(\begin{array}{cc}
a x & b x+y c \\
0 & c z
\end{array}\right)=\mathbf{0} .
$$

Suppose (3.1) holds. Then $x=z=0$, so $a y \equiv 0(\bmod n)$, a contradiction. Likewise, if (3.2) holds, then once again $x=z=0$, so $c y \equiv 0(\bmod n)$, a contradiction. Hence, we must have either $\operatorname{gcd}(a, n) \neq 1$ or $\operatorname{gcd}(c, n) \neq 1$.

Now, suppose $\operatorname{gcd}(a, n) \neq 1$, and let $s_{1}=n / \operatorname{gcd}(a, n)$. Then

$$
\left(\begin{array}{ll}
a & b \\
0 & c
\end{array}\right)\left(\begin{array}{cc}
s_{1} & s_{1} \\
0 & 0
\end{array}\right)=\mathbf{0}
$$

so $A \in Z_{2}(n)$. On the other hand, suppose that $\operatorname{gcd}(a, n)=1$ but $\operatorname{gcd}(c, n) \neq$ 1 , and let $s_{2}=n / \operatorname{gcd}(c, n)$. Also, let $y$ be the solution of $a y+b s_{2} \equiv 0$ $(\bmod n)$. Then

$$
\left(\begin{array}{ll}
a & b \\
0 & c
\end{array}\right)\left(\begin{array}{cc}
0 & y \\
0 & s_{2}
\end{array}\right)=\left(\begin{array}{cc}
0 & a y+b s_{2} \\
0 & c s_{2}
\end{array}\right)=\mathbf{0},
$$

so $A \in Z_{2}(n)$.

Using Lemma 3.1, it is easy to show that if $n \geq 2$, then

$$
\left|Z_{2}(n)\right|=n^{3}-n \phi(n)^{2}-1,
$$

where $\phi$ denotes Euler's totient function. In particular,

$$
\left|Z_{2}(p)\right|=2 p^{2}-p-1
$$

for every prime $p$. To establish (3.3), first note that $\left|T_{2}(n)-\{\mathbf{0}\}\right|=n^{3}-1$. By Lemma 3.1, $\left|Z_{2}(n)\right|=n^{3}-1-n m^{2}$, where $m$ is the number of elements $k \in \mathbb{Z}_{n}$ such that $\operatorname{gcd}(k, n)=1$; but this value is precisely $\phi(n)$, so the 


\section{T. FENSTERMACHER AND E. GEGNER}

result is established. It then follows from the definition of $\tilde{\Gamma}\left(T_{2}(n)\right)$ that $\left|\tilde{\Gamma}\left(T_{2}(n)\right)\right|=n^{3}-n \phi(n)^{2}-1$ as well. It is clear that $\left|\bar{\Gamma}\left(T_{2}(n)\right)\right| \leq\left|\Gamma\left(T_{2}(n)\right)\right|$ for all $n \geq 2$. Theorem 3.12 will show that, in fact, equality holds.

In the following definitions $G$ denotes an arbitrary directed or undirected graph.

Definition 3.2. The graph $G$ is connected if for any distinct vertices $v_{1}, v_{2}$ of $G$, there exists a path in $G$ from $v_{1}$ to $v_{2}$.

Definition 3.3. For each pair of vertices $u, v$ of $G$, let $P_{s}(u, v)$ denote the shortest path from $u$ to $v$ for all vertices $u, v$ of $G$. Then the diameter of $G$, denoted by $d(G)$, is

$$
d(G)=\max \left\{P_{s}(u, v): u, v \text { vertices of } G\right\} .
$$

We use the following result, due to Redmond [13], to establish several properties of $\Gamma\left(T_{2}(n)\right)$.

Theorem 3.4. Let $R$ be a ring. Then $\Gamma(R)$ is connected if and only if $Z^{L}(R)=Z^{R}(R)$. Moreover, if $\Gamma(R)$ is connected, then $d(\Gamma(R)) \leq 3$.

Corollary 3.5. Let $n \geq 2$. Then $\Gamma\left(T_{2}(n)\right)$ is connected.

Proof. Suppose $A=\left(\begin{array}{cc}a_{0} & b_{0} \\ 0 & c_{0}\end{array}\right)$ is an element of $Z\left(T_{2}(n)\right)$. Let

$$
\begin{aligned}
a_{1} & \equiv \frac{n}{\operatorname{gcd}\left(a_{0}, n\right)} \quad(\bmod n) \\
b_{1} & \equiv \frac{-b_{0} n}{\operatorname{gcd}\left(c_{0}, n\right)} \quad(\bmod n) \\
c_{1} & \equiv \frac{a_{0} n}{\operatorname{gcd}\left(c_{0}, n\right)} \quad(\bmod n) \\
a_{2} & \equiv \frac{c_{0} n}{\operatorname{gcd}\left(a_{0}, n\right)} \quad(\bmod n) \\
b_{2} & \equiv \frac{-b_{0} n}{\operatorname{gcd}\left(a_{0}, n\right)} \quad(\bmod n) \\
c_{2} & \equiv \frac{n}{\operatorname{gcd}\left(c_{0}, n\right)} \quad(\bmod n)
\end{aligned}
$$

and let

$$
B_{1}=\left(\begin{array}{cc}
a_{1} & b_{1} \\
0 & c_{1}
\end{array}\right) \quad B_{2}=\left(\begin{array}{cc}
a_{2} & b_{2} \\
0 & c_{2}
\end{array}\right) .
$$

Then $A B_{1}=\mathbf{0}$ and $B_{2} A=\mathbf{0}$. By Lemma 3.1, either $\operatorname{gcd}\left(a_{0}, n\right) \neq 1$ or $\operatorname{gcd}\left(c_{0}, n\right) \neq 1$. Consequently, $a_{1}=0$ implies $c_{1} \neq 0$ and $c_{2}=0$ implies $a_{2} \neq 0$, so $B_{1} \neq \mathbf{0}$ and $B_{2} \neq \mathbf{0}$. Therefore, $A \in Z^{L}(n)$ and $A \in Z^{R}(n)$, and the result follows by Theorem 3.4. 


\section{ZERO-DIVISOR GRAPHS OF $2 \times 2$ MATRIX RINGS OVER $\mathbb{Z}_{n}$}

Theorem 3.6. Let $n \geq 2$. If $n$ is not a prime power, then there is no element $A \in Z_{2}(n)$ such that $A$ is adjacent to all $B \in Z_{2}(n)$ in $\tilde{\Gamma}\left(T_{2}(n)\right)$. If $n=p^{k}$ for some prime $p$ and $k \in \mathbb{N}$, then $A \in Z_{2}(n)$ is adjacent to every other vertex in $\tilde{\Gamma}\left(T_{2}(n)\right)$ if and only if $A=\left(\begin{array}{cc}0 & s p^{k-1} \\ 0 & 0\end{array}\right)$ for some $s$ relatively prime to $n$.

Proof. Let $n=p_{1}^{e_{1}} p_{2}^{e_{2}} \cdots p_{m}^{e_{m}}$, where the $p_{i}$ are prime and $n$ is not a prime power. Suppose that

$$
X=\left(\begin{array}{ll}
x & y \\
0 & z
\end{array}\right) \in Z_{2}(n)
$$

is adjacent to every other vertex in $\Gamma\left(T_{2}(n)\right)$; that is, $X A=\mathbf{0}$ or $A X=\mathbf{0}$ for all $A \in Z_{2}(n)$. Choosing $A=\left(\begin{array}{cc}p_{1} & 1 \\ 0 & 1\end{array}\right)$ and then $A=\left(\begin{array}{cc}1 & 1 \\ 0 & p_{1}\end{array}\right)$ shows that $x=z=0$. Thus $y \neq 0$, and since $n$ is not a prime power, there exists some $j \in \mathbb{N}, 1 \leq j \leq m$ such that $n \nmid p_{j} y$. Let $B=\left(\begin{array}{cc}1 & 1 \\ 0 & p_{j}\end{array}\right)$. Then

$$
X B=\left(\begin{array}{cc}
0 & y p_{j} \\
0 & 0
\end{array}\right)=\mathbf{0} \quad \text { or } \quad B X=\left(\begin{array}{ll}
0 & y \\
0 & 0
\end{array}\right)=\mathbf{0} .
$$

In either case, $y=0$, a contradiction.

To prove the second part of the theorem, let $n=p^{k}$, where $p$ is prime and $k \in \mathbb{N}$; let $s \in \mathbb{N}$ be relatively prime to $n$; and let

$$
A_{1}=\left(\begin{array}{cc}
0 & s p^{k-1} \\
0 & 0
\end{array}\right) \text {. }
$$

By Lemma 3.1, if $C=\left(\begin{array}{ll}x & y \\ 0 & z\end{array}\right) \in Z_{2}(n)$, then either $p \mid x$ or $p \mid z$. If $p \mid z$, then $A_{1} C=\mathbf{0}$, and if $p \mid x$, then $C A_{1}=\mathbf{0}$.

Conversely, let $A_{2} \in Z_{2}(n)$, and suppose that $A_{2}$ is adjacent to every other vertex in $\Gamma\left(T_{2}(n)\right)$. Once again $A_{2}$ must have the form

$$
A_{2}=\left(\begin{array}{cc}
0 & y \\
0 & 0
\end{array}\right)
$$

Let $B=\left(\begin{array}{ll}1 & 1 \\ 0 & p\end{array}\right)$, and suppose $y \neq s p^{k-1}$ for all $s$ relatively prime to $n$. Then either

$$
A_{2} B=\left(\begin{array}{cc}
0 & y p \\
0 & 0
\end{array}\right)=\mathbf{0} \quad \text { or } \quad B A_{2}=\left(\begin{array}{cc}
0 & y \\
0 & 0
\end{array}\right)=\mathbf{0},
$$

both of which imply that $y=0$, a contradiction.

MISSOURI J. OF MATH. SCI., FALL 2014 


\section{T. FENSTERMACHER AND E. GEGNER}

Theorem 3.7. Let $n \geq 2$. Then $d\left(\tilde{\Gamma}\left(T_{2}(n)\right)\right)=2$ if and only if $n$ is a prime power.

Proof. Suppose $n$ is a prime power, and let $X, Y \in Z_{2}(n)$. Then by Theorem 3.6 there is a matrix $A \in Z_{2}(n)$ such that $X \rightarrow A \rightarrow Y$ is a path in $\tilde{\Gamma}\left(T_{2}(n)\right)$. By Theorem 3.6, the diameter is greater than 1 , so the result follows.

Now suppose that $n$ is not a prime power, and let $p, q$ be distinct prime divisors of $n$. Consider the matrices

$$
A=\left(\begin{array}{cc}
p & 0 \\
0 & 1
\end{array}\right), B=\left(\begin{array}{ll}
q & 0 \\
0 & 1
\end{array}\right)
$$

in $Z_{2}(n)$. If there is some $M=\left(\begin{array}{cc}m_{1} & m_{2} \\ 0 & m_{3}\end{array}\right)$ such that $A \rightarrow M \rightarrow B$ is a path in $\tilde{\Gamma}\left(T_{2}(n)\right)$, then one of $A M, M A$ is $\mathbf{0}$ and one of $B M, M B$ is 0. Multiplying out $A M, M A, B M$ and $M B$ shows that in each of the four possible cases $m_{1}=m_{2}=m_{3}=0$. Thus, $d\left(\tilde{\Gamma}\left(T_{2}(n)\right)\right) \neq 2$.

Definition 3.8. Let $p$ be prime. Then for each $1 \leq k \leq p$ we define the following subsets of $Z_{2}(p)$ :

$$
\begin{aligned}
& \mathcal{S}_{0}(p):=\left\{\left(\begin{array}{ll}
0 & b \\
0 & 0
\end{array}\right): b \in \mathbb{Z}_{p}, b \neq 0\right\} \\
& \mathcal{S}_{k}(p):=\left\{\left(\begin{array}{cc}
a & k a \\
0 & 0
\end{array}\right): a \in \mathbb{Z}_{p}, a \neq 0\right\} \\
& \mathcal{S}_{k}^{\prime}(p):=\left\{\left(\begin{array}{cc}
0 & -k c \\
0 & c
\end{array}\right): c \in \mathbb{Z}_{p}, c \neq 0\right\} .
\end{aligned}
$$

When the ring $\mathbb{Z}_{p}$ is clear from the context, we will often write $\mathcal{S}_{k}(p)$ and $\mathcal{S}_{k}^{\prime}(p)$ as $\mathcal{S}_{k}$ and $\mathcal{S}_{k}^{\prime}$, respectively.

Lemma 3.9. If $p$ is prime, then the set $\left\{\mathcal{S}_{0}, \mathcal{S}_{1}, \ldots, \mathcal{S}_{p}, \mathcal{S}_{1}^{\prime}, \ldots, \mathcal{S}_{p}^{\prime}\right\}$ partitions $Z_{2}(p)$.

Proof. Clearly the intersection of any two of the sets is empty. Let $A=$ $\left(\begin{array}{ll}a & b \\ 0 & c\end{array}\right) \in Z_{2}(p)$. Since $p$ is prime, Lemma 3.1 implies that $a=0$ or $c=0$. If $c=0$ and $a \neq 0$, then we must have $b \equiv k a(\bmod p)$ for some $1 \leq k \leq p$, so $A \in \mathcal{S}_{k}$. Likewise, if $a=0$ and $c \neq 0$, then $b \equiv-k c(\bmod p)$ for some $1 \leq k \leq p$, so $A \in \mathcal{S}_{k}^{\prime}$. Finally, if $a=c=0$, then $A \in \mathcal{S}_{0}$. Thus, $\bigcup_{k=0}^{p} \mathcal{S}_{k} \cup \bigcup_{k=1}^{p} \mathcal{S}_{k}^{\prime}=Z_{2}(p)$.

Let $n \geq 2$, and let $A_{1}, A_{2}, \ldots, A_{k}$ be distinct vertices in $Z_{2}(n)$. If $A_{i} A_{i+1}=\mathbf{0}$ for each $1 \leq i<k$, then $A_{1} \rightarrow A_{2} \rightarrow \cdots \rightarrow A_{k}$ denotes 
the path from $A_{1}$ to $A_{k}$ in $\Gamma\left(T_{2}(n)\right)$ via the vertices $A_{i}$, where each vertex is visited exactly once. Moreover, if $B_{1}, B_{2} \subseteq Z_{2}(n)$, then we write $B_{1} \rightrightarrows B_{2}$ if $B B^{\prime}=\mathbf{0}$ for all $B \in B_{1}, B^{\prime} \in B_{2}$, and we write $B_{1} \perp B_{2}$ if $B B^{\prime} \neq \mathbf{0}$ for all $B \in B_{1}, B^{\prime} \in B_{2}$. The following lemma can easily be verified using direct calculation and the definition of the sets $\mathcal{S}_{0}, \mathcal{S}_{k}, \mathcal{S}_{k}^{\prime}$.

Lemma 3.10. Let $p$ be prime. If $k, j \in\{1,2, \ldots, p\}$, then

$$
\begin{array}{ll}
\mathcal{S}_{0} \rightrightarrows \mathcal{S}_{0}, \mathcal{S}_{k} & \\
\mathcal{S}_{0} \perp \mathcal{S}_{j}^{\prime} & \\
\mathcal{S}_{j}^{\prime} \rightrightarrows \mathcal{S}_{0}, \mathcal{S}_{k} & \\
\mathcal{S}_{j}^{\prime} \perp \mathcal{S}_{k}^{\prime} & \\
\mathcal{S}_{k} \rightrightarrows \mathcal{S}_{j}^{\prime} & \text { if } k=j \\
\mathcal{S}_{k} \perp \mathcal{S}_{j}^{\prime} & \text { if } k \neq j \\
\mathcal{S}_{k} \perp \mathcal{S}_{0}, \mathcal{S}_{j} &
\end{array}
$$

where $\mathcal{S}_{0}, \mathcal{S}_{k}, \mathcal{S}_{j}, \mathcal{S}_{k}^{\prime}, \mathcal{S}_{j}^{\prime} \subseteq Z_{2}(p)$.

It follows from Lemma 3.10 that a matrix $A \in Z_{2}(p)$ is nilpotent if and only if $A \in \mathcal{S}_{0}$. Figure 1 depicts the relationships given in Lemma 3.10. If $A, B \in\left\{\mathcal{S}_{0}, \mathcal{S}_{1}, \ldots, \mathcal{S}_{p}, \mathcal{S}_{1}^{\prime}, \ldots, \mathcal{S}_{p}^{\prime}\right\}$, then there is an arrow going from $A$ to $B$ if and only if $A \rightrightarrows B$. Moreover, the absence of an arrow from $A$ to $B$ implies that $A \perp B$. Some of the lines are dashed in order to make the diagram more readable. We call this the $\mathcal{S}, \mathcal{S}^{\prime}$ diagram.

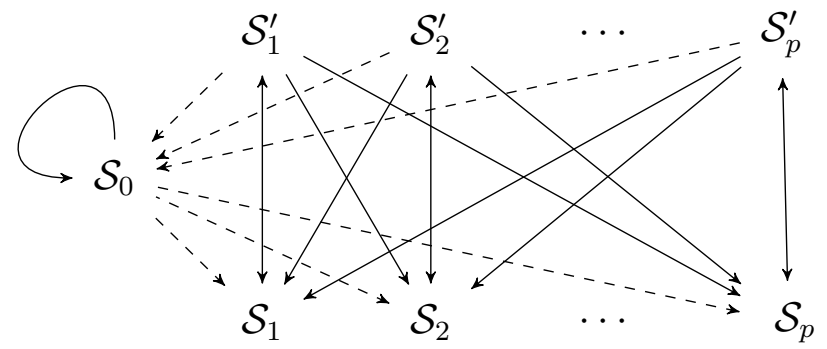

Figure 1. The $\mathcal{S}, \mathcal{S}^{\prime}$ Diagram.

Since the $\mathcal{S}, \mathcal{S}^{\prime}$ diagram portrays every conclusion of Lemma 3.10, when Lemma 3.10 is cited it is almost always more practical to refer to the diagram rather than the lemma itself.

The proof of the following theorem is an excellent application of the $\mathcal{S}, \mathcal{S}^{\prime}$ diagram.

Theorem 3.11. Let $p$ be prime. Then $\chi\left(\Gamma\left(T_{2}(p)\right)\right)=p+1$.

MISSOURI J. OF MATH. SCI., FALL 2014 


\section{T. FENSTERMACHER AND E. GEGNER}

Proof. Suppose we have colored the graph so that no two adjacent vertices have the same color. Since $\mathcal{S}_{0} \rightrightarrows \mathcal{S}_{0}$, every element in $\mathcal{S}_{0}$ must have a different color, so $\chi\left(\Gamma\left(T_{2}(p)\right)\right) \geq p-1$. Since $\mathcal{S}_{k}^{\prime} \rightrightarrows \mathcal{S}_{0}$ and $\mathcal{S}_{0} \rightrightarrows \mathcal{S}_{k}$, any vertex $v$ of $\Gamma\left(T_{2}(p)\right)$ such that $v \notin \mathcal{S}_{0}$ does not have a color found in $\mathcal{S}_{0}$. Moreover, since $\mathcal{S}_{k}^{\prime} \rightrightarrows \mathcal{S}_{k}$ for each $k \in\{1, \ldots, p\}$, it follows that if $a \in \mathcal{S}_{k}, b \in \mathcal{S}_{k}^{\prime}$, then $a$ and $b$ must be colored with different colors. Thus, $\chi\left(\Gamma\left(T_{2}(p)\right)\right) \geq p+1$. It is easy to verify that choosing a different color for each of the $p-1$ elements of $\mathcal{S}_{0}$, choosing a $p$ th distinct color for the elements of $\bigcup_{k=1}^{p} \mathcal{S}_{k}$, and choosing a $(p+1)$ th color for the elements of $\bigcup_{k=1}^{p} \mathcal{S}_{k}^{\prime}$ gives a valid coloring of the graph.

In the proof of Theorem 3.12 will shall use the following notation: Let $n \geq 2$, let $p$ be a factor of $n$, and suppose

$$
X=\left(\begin{array}{cc}
x_{1} & x_{2} \\
0 & x_{3}
\end{array}\right) \in Z_{2}(n) .
$$

Then we define the element $X_{k} \in T_{2}(k)$ by

$$
X_{k}=\left(\begin{array}{cc}
x_{1}^{\prime} & x_{2}^{\prime} \\
0 & x_{3}^{\prime}
\end{array}\right)
$$

where $x_{i}^{\prime} \equiv x_{i}(\bmod k)$ for each $i \in\{1,2,3\}$.

Theorem 3.12. Let $n \geq 2$, and let $X=\left(\begin{array}{ll}a & b \\ 0 & c\end{array}\right) \in Z_{2}(n)$. Then $\operatorname{deg}(X)>0$ in $\bar{\Gamma}\left(T_{2}(n)\right)$.

Proof. Write $n=p_{1}^{e_{1}} \cdots p_{m}^{e_{m}}$, where the $p_{i}$ are distinct primes. Suppose that there exists some prime divisor $p$ of $n$ that divides $a, b$, and $c$. Renaming if necessary, we may assume $p=p_{1}$. Let

$$
Y=\left(\begin{array}{cc}
p_{1}^{e_{1}-1} p^{e_{2}} \cdots p_{m}^{e_{m}} & p_{1}^{e_{1}-1} p^{e_{2}} \cdots p_{m}^{e_{m}} \\
0 & p_{1}^{e_{1}-1} p^{e_{2}} \cdots p_{m}^{e_{m}}
\end{array}\right) .
$$

Then $Y \in Z_{2}(n)$ and $X Y=Y X=\mathbf{0}$ in $\mathbb{Z}_{n}$.

On the other hand, suppose that no divisor of $n$ divides $a, b$, and $c$ simultaneously. By Lemma 3.1, there is a prime divisor $q$ of $n$ that divides either $a$ or $c$. Then $X_{q} \in Z_{2}(q)$, and by Lemma 3.10 we see that there exists some $W^{\prime} \in Z_{2}(q)$ such that $X_{q} W^{\prime}=W^{\prime} X_{q}=\mathbf{0}$ in $\mathbb{Z}_{q}$. We claim that $X W=W X=\mathbf{0}$, where $W=\left(\frac{n}{q}\right) W^{\prime} \in Z_{2}(n)$. To see this, write $X=q M+X_{q}$, where $M \in T_{2}(n)$. Then $X W=\left(q M+X_{q}\right)\left(\frac{n}{q}\right) W^{\prime}=$ $n M W^{\prime}+\left(\frac{n}{q}\right) X_{q} W^{\prime}=\mathbf{0}$ in $\mathbb{Z}_{n}$. Similarly, $W X=\mathbf{0}$ in $\mathbb{Z}_{n}$, and the proof is complete. 


\section{ZERO-DIVISOR GRAPHS OF $2 \times 2$ MATRIX RINGS OVER $\mathbb{Z}_{n}$}

Definition 3.13. Let $G$ be a connected graph. Then the edge connectivity of $G$, denoted by $\kappa(G)$, is the smallest number of edges that can be removed from $G$ to disconnect the graph.

Remark. Let $n=p_{1}^{e_{1}} \cdots p_{m}^{e_{m}}$, where the $p_{i}$ are prime and $p_{1}<p_{2}<\cdots<$ $p_{m}$. If $A=\left(\begin{array}{cc}a_{1} & a_{2} \\ 0 & a_{3}\end{array}\right) \in Z_{2}(n)$, then the matrices $A, 2 A, \ldots,\left(p_{1}-1\right) A$ are all distinct elements of $Z_{2}(n)$. For suppose there exist integers $k_{1}, k_{2} \in$ $\left\{1, \ldots, p_{1}-1\right\}$ such that $k_{1} A=k_{2} A$. Then $\left(k_{1}-k_{2}\right) a_{i} \equiv 0(\bmod n)$ for each $i \in\{1,2,3\}$. Let $s \in\{1,2,3\}$ such that $a_{s} \neq 0$. Then since $a_{s}<n$ and $\left|k_{1}-k_{2}\right|<p_{1}$, it follows that $k_{1}=k_{2}$.

Theorem 3.14. Let $n=p_{1}^{e_{1}} \cdots p_{m}^{e_{m}}$, with $p_{1}<p_{2}<\cdots<p_{m}$. Then $\Gamma\left(T_{2}(n)\right)$ has edge connectivity $p_{1}-1$.

Proof. Let $k=p_{1}^{e_{1}-1} p_{2}^{e_{2}} \cdots p_{m}^{e_{m}}$, let

$$
X=\left(\begin{array}{ll}
x & y \\
0 & z
\end{array}\right) \in Z_{2}(n),
$$

and consider the element

$$
A=\left(\begin{array}{cc}
1 & k \\
0 & p_{1}
\end{array}\right) \in Z_{2}(n)
$$

Then

$$
A X=\left(\begin{array}{cc}
x & y+k z \\
0 & p_{1} z
\end{array}\right)=\mathbf{0}
$$

if and only if $x=0, z=r k$ where $\operatorname{gcd}\left(r, p_{1}\right)=1$, and $y=-k z$. Thus,

$$
\kappa\left(\Gamma\left(T_{2}(n)\right)\right) \leq p_{1}-1 .
$$

Now, suppose $M=\left\{m_{i j}\right\}, N=\left\{n_{i j}\right\} \in Z_{2}(n)$, and let $E$ be any set of $p_{1}-2$ edges in $\Gamma\left(T_{2}(n)\right)$. To prove $\kappa\left(\Gamma\left(T_{2}(n)\right)\right) \geq p_{1}-1$, it suffices to show that there is a path from $M$ to $N$ in $\Gamma\left(T_{2}(n)\right)$ that contains no edge in $E$. By Lemma 3.1, there exist prime factors $p, q$ of $n$ such that $p$ divides at least one of $m_{11}, m_{22}$ and $q$ divides at least one of $n_{11}, n_{22}$. Define

$$
\begin{aligned}
S_{1} & =\left\{\left(\begin{array}{ll}
0 & x \\
0 & 0
\end{array}\right) \in Z_{2}(n): x \in\langle n / p\rangle\right\} \\
S_{2} & =\left\{\left(\begin{array}{ll}
0 & y \\
0 & x
\end{array}\right) \in Z_{2}(n): x \in\langle n / p\rangle \text { and } m_{11} y+m_{12} x \equiv 0 \quad(\bmod n)\right\} \\
R_{1} & =\left\{\left(\begin{array}{ll}
0 & x \\
0 & 0
\end{array}\right) \in Z_{2}(n): x \in\langle n / q\rangle\right\} \\
R_{2} & =\left\{\left(\begin{array}{ll}
x & y \\
0 & 0
\end{array}\right) \in Z_{2}(n): x \in\langle n / q\rangle \text { and } n_{12} x+n_{22} y \equiv 0 \quad(\bmod n)\right\}
\end{aligned}
$$

MISSOURI J. OF MATH. SCI., FALL 2014 


\section{T. FENSTERMACHER AND E. GEGNER}

Clearly $\left|S_{1}\right|=p-1$ and $\left|R_{1}\right|=q-1$. Also, note that if $p \nmid m_{11}$, then $\left|S_{2}\right| \geq p-1$, and if $q \nmid n_{22}$, then $\left|R_{2}\right| \geq q-1$. Define $s=1$ if $p \mid m_{11}$, $s=2$ otherwise. Similarly, define $r=1$ if $q \mid n_{22}, r=2$ otherwise. Note that $M V=\mathbf{0}$ for all $V \in S_{s}$, and $W N=\mathbf{0}$ for all $W \in R_{r}$. Let $a=\min \{p-1, q-1\}$. Let $V_{1}, \ldots, V_{a}$ be distinct elements of $S_{s}$, and let $W_{1}, \ldots, W_{a}$ be distinct elements of $R_{r}$ such that $W_{i}=V_{i}$ whenever $W_{i} \in\left\{V_{1}, \ldots, V_{a}\right\}$. Then

$$
K=\left\{M \rightarrow V_{i} \rightarrow W_{i} \rightarrow N: i \in\{1, \ldots, a\}\right\}
$$

is a set of $a \geq p_{1}-1$ paths in $\Gamma\left(T_{2}(n)\right)$, no two of which share an edge. Thus, there is a path in $K$ that contains no edge of $E$. Consequently,

$$
\kappa\left(\Gamma\left(T_{2}(n)\right)\right) \geq p_{1}-1,
$$

so $\kappa\left(\Gamma\left(T_{2}(n)\right)\right)=p_{1}-1$ by equation (3.4).

The proof of our next theorem mimics that of Theorem 3.14.

Theorem 3.15. Let $n=p_{1}^{e_{1}} \cdots p_{m}^{e_{m}}$, where the $p_{i}$ are prime and $p_{1}<p_{2}<$ $\cdots<p_{m}$. Then $\tilde{\Gamma}\left(T_{2}(n)\right)$ has edge connectivity $p_{1}^{2}-1$.

Proof. Let $k=p_{1}^{e_{1}-1} p_{2}^{e_{2}} \cdots p_{m}^{e_{m}}$, let

$$
X=\left(\begin{array}{ll}
x & y \\
0 & z
\end{array}\right) \in Z_{2}(n),
$$

and consider the element

$$
A=\left(\begin{array}{cc}
1 & k \\
0 & p_{1}
\end{array}\right) \in Z_{2}(n)
$$

Then

$$
A X=\left(\begin{array}{cc}
x & y+k z \\
0 & p_{1} z
\end{array}\right)=\mathbf{0}
$$

if and only if $x=0, z=r k$ where $\operatorname{gcd}\left(r, p_{1}\right)=1$, and $y \equiv-k z(\bmod n)$. On the other hand,

$$
X A=\left(\begin{array}{cc}
x & k x+p_{1} y \\
0 & p_{1} z
\end{array}\right)=\mathbf{0}
$$

if and only if $x=0$, and $z=r_{1} k, y=r_{2} k$ for some $r_{1}, r_{2} \in\left\{0,1, \ldots, p_{1}-1\right\}$ where $r_{1}$ and $r_{2}$ are not both zero. Hence, if $A X=\mathbf{0}$, then $X A=\mathbf{0}$, and $\operatorname{deg}(A)=p_{1}^{2}-1$. Thus,

$$
\kappa\left(\tilde{\Gamma}\left(T_{2}(n)\right)\right) \leq p_{1}^{2}-1
$$

Now, let $E$ be any collection of $p_{1}^{2}-2$ edges of $\tilde{\Gamma}\left(T_{2}(n)\right)$, and suppose $M=\left\{m_{i j}\right\}, N=\left\{n_{i j}\right\} \in Z_{2}(n)$. To prove that $\kappa\left(\tilde{\Gamma}\left(T_{2}(n)\right)\right) \geq p_{1}^{2}-1$ is suffices to show that there exists a path from $M$ to $N$ in $\tilde{\Gamma}\left(T_{2}(n)\right)$ that 


\section{ZERO-DIVISOR GRAPHS OF $2 \times 2$ MATRIX RINGS OVER $\mathbb{Z}_{n}$}

contains no edge in $E$. By Lemma 3.1, there exist prime factors $p, q$ of $n$ such that $p$ divides at least one of $m_{11}, m_{22}$ and $q$ divides at least one of $n_{11}, n_{22}$. Define

$$
\begin{aligned}
S_{1} & =\left\{\left(\begin{array}{ll}
x & y \\
0 & 0
\end{array}\right) \in Z_{2}(n): x, y \in\langle n / p\rangle\right\} \\
S_{2} & =\left\{\left(\begin{array}{ll}
0 & x \\
0 & y
\end{array}\right) \in Z_{2}(n): x, y \in\langle n / p\rangle\right\} \\
R_{1} & =\left\{\left(\begin{array}{ll}
x & y \\
0 & 0
\end{array}\right) \in Z_{2}(n): x, y \in\langle n / q\rangle\right\} \\
R_{2} & =\left\{\left(\begin{array}{ll}
0 & x \\
0 & y
\end{array}\right) \in Z_{2}(n): x, y \in\langle n / q\rangle\right\} .
\end{aligned}
$$

Note that $\left|S_{1}\right|=\left|S_{2}\right|=p^{2}-1 \geq p_{1}^{2}-1$ and $\left|R_{1}\right|=\left|R_{2}\right|=q^{2}-1 \geq p_{1}^{2}-1$. Let $s, r \in\{1,2\}$ such that $p$ divides $m_{s s}$ and $q$ divides $n_{r r}$. Note that for all $V \in S_{s}$, either $M V=\mathbf{0}$ or $V M=\mathbf{0}$, and for all $W \in R_{r}$, either $N W=\mathbf{0}$ or $W N=\mathbf{0}$. Let $a=\min \left\{p^{2}-1, q^{2}-1\right\}$. Let $V_{1}, \ldots, V_{a}$ be distinct matrices in $S_{s}$, and let $W_{1}, \ldots, W_{a}$ be distinct matrices in $R_{r}$, where $W_{i}=V_{i}$ whenever $W_{i} \in\left\{V_{1}, \ldots, V_{a}\right\}$. Then

$$
K=\left\{M \rightarrow V_{i} \rightarrow W_{i} \rightarrow N: i \in\{1, \ldots, a\}\right\}
$$

is a set of $a \geq p_{1}^{2}-1$ paths in $\tilde{\Gamma}\left(T_{2}(n)\right)$, no two of which share an edge. Thus, there is a path in $K$ that contains no edge of $E$. Therefore $\kappa\left(\Gamma\left(T_{2}(n)\right)\right) \geq$ $p_{1}^{2}-1$, and it follows from (3.5) that $\kappa\left(\Gamma\left(T_{2}(n)\right)\right)=p_{1}^{2}-1$.

Definition 3.16. A graph $G$ with $n$ vertices is Hamiltonian if $G$ contains a cycle of length $n$. $G$ is pancyclic if $G$ contains a cycle of all lengths $\ell$, for $3 \leq \ell \leq n$. $G$ is vertex-pancyclic if each vertex in $G$ belongs to a cycle of every length $3 \leq \ell \leq n$.

If $\mathcal{P}_{1}, \mathcal{P}_{2}$ are paths in $\Gamma\left(T_{2}(n)\right)$, and the last vertex in $\mathcal{P}_{1}$ is adjacent to the first vertex of $\mathcal{P}_{2}$, then $\mathcal{P}_{1}+\mathcal{P}_{2}$ denotes the path formed by appending $\mathcal{P}_{2}$ to the end of $\mathcal{P}_{1}$. In a slight abuse of notation, if $A$ is a vertex of $\Gamma\left(T_{2}(n)\right)$ and $A$ is adjacent to the last element of $\mathcal{P}_{1}$, then $\mathcal{P}_{1}+A$ denotes the path formed by appending $A$ to the end of $\mathcal{P}_{1}$.

Remark. Note that $\Gamma\left(T_{2}(2)\right)$ is pancyclic but not vertex-pancyclic since no element in $\mathcal{S}_{0}$ can be in a cycle of length four. This can be visually verified using the $\mathcal{S}, \mathcal{S}^{\prime}$ diagram.

Theorem 3.17. $\Gamma\left(T_{2}(n)\right)$ is vertex-pancyclic if and only if $n$ is an odd prime.

Proof. The remark preceding the theorem takes care of the case $n=2$. Suppose $n$ is an odd prime. For $i \in\{0, \ldots, n\}, j \in\{1, \ldots, n\}$, enumerate 


\section{T. FENSTERMACHER AND E. GEGNER}

the elements of $\mathcal{S}_{i}$ and $\mathcal{S}_{j}^{\prime}$ :

$$
\begin{aligned}
\mathcal{S}_{i} & =\left\{S_{i, 1}, \ldots, S_{i, n-1}\right\} \\
\mathcal{S}_{j}^{\prime} & =\left\{S_{j, 1}, \ldots, S_{j, n-1}\right\} .
\end{aligned}
$$

For $1 \leq k \leq n$, even $2 \leq e \leq 2 n$, and odd $3 \leq d \leq 2 n+1$, define

$$
\begin{aligned}
\mathcal{P}_{k, e, i}:= & S_{k, i}^{\prime} \rightarrow S_{k+1, i} \rightarrow S_{k+1, i}^{\prime} \rightarrow \cdots \rightarrow S_{k+\frac{e}{2}-1, i} \rightarrow S_{k+\frac{e}{2}-1, i}^{\prime} \rightarrow S_{k, i} \\
\mathcal{P}_{k, d, i}:= & S_{k, i}^{\prime} \rightarrow S_{0, i} \rightarrow S_{k+1, i} \rightarrow S_{k+1, i}^{\prime} \rightarrow \cdots \rightarrow S_{k+\left\lfloor\frac{d}{2}\right\rfloor-1, i} \rightarrow S_{k+\left\lfloor\frac{d}{2}\right\rfloor-1, i}^{\prime} \\
& \rightarrow S_{k, i}
\end{aligned}
$$

where we take $S_{m, i}=S_{m-n, i}, S_{m, i}^{\prime}=S_{m-n, i}^{\prime}$ whenever $m>n$. The $\mathcal{S}, \mathcal{S}^{\prime}$ diagram shows that for any $3 \leq \ell \leq 2 n+1, \mathcal{P}_{k, \ell, i}$ is a path of length $\ell-1$ in $\Gamma\left(T_{2}(n)\right)$.

Let $3 \leq \ell \leq\left|Z_{2}(n)\right|=2 n^{2}-n-1$. We must show that for each $S \in Z_{2}(n), \Gamma\left(T_{2}(n)\right)$ contains a cycle of length $\ell$ that passes through $S$. If $\ell \leq 2 n+1$, then $\mathcal{P}_{k, \ell, i}+S_{k, i}^{\prime}$ is a cycle of length $\ell$ that contains $S_{k, i}^{\prime}$ and $S_{k, i}$. If $2 n+2 \leq \ell \leq 2 n^{2}-2 n+1$, then there is some $t \in\{1, \ldots, n-2\}$ such that $2(t n+1) \leq \ell \leq 2(t+1) n+1$, and

$$
\mathcal{P}_{k, 2 n, i}+\mathcal{P}_{k, 2 n, i+1}+\cdots+\mathcal{P}_{k, 2 n, i+t-1}+\mathcal{P}_{k, \ell-2 t n, i+t}+S_{k, i}^{\prime}
$$

is a cycle in $\Gamma\left(T_{2}(n)\right)$ with length $(2 n-1)(t)+t+(\ell-2 t n-1)+1=\ell$, where we take $\mathcal{P}_{k, l, m}=\mathcal{P}_{k, l, m-n+1}$ whenever $m>n-1$. If $2((n-1) n+1) \leq \ell<$ $(2 n+1)(n-1)$, then

$$
\begin{aligned}
\mathcal{P}_{k, 2 n+1, i}+\mathcal{P}_{k, 2 n+1, i+1} & +\cdots+\mathcal{P}_{k, 2 n+1, i+\ell-2 n(n-1)-1}+\mathcal{P}_{k, 2 n, i+\ell-2 n(n-1)} \\
& +\cdots+\mathcal{P}_{k, 2 n, i+n-2}+S_{k, i}^{\prime}
\end{aligned}
$$

is a cycle of length $(2 n+1)(\ell-2 n(n-1))+2 n\left(2 n^{2}-n-1-\ell\right)=\ell$. Finally, if $\ell=(2 n+1)(n-1)$, then

$$
\mathcal{P}_{k, 2 n+1, i}+\mathcal{P}_{k, 2 n+1, i+1}+\cdots+\mathcal{P}_{k, 2 n+1, i+n-2}+S_{k, i}^{\prime}
$$

is a cycle of length $\ell$. This shows that $\Gamma\left(T_{2}(n)\right)$ is pancyclic. Moreover, notice that each cycle given above passes through $S_{k, i}^{\prime}$ and $S_{k, i}$ for all $\ell$, and $S_{0, i}$ if $\ell$ is odd. Since this holds for all $k \in\{1, \ldots, n\}$ and $i \in\{1, \ldots, n-1\}$, we have that for each $S \in Z_{2}(n)$ and $3 \leq \ell \leq\left|Z_{2}(n)\right|, \Gamma\left(T_{2}(n)\right)$ contains a cycle of length $\ell$ containing $S$ unless $S \in \mathcal{S}_{0}$ and $\ell$ is even.

Thus, to prove that $\Gamma\left(T_{2}(n)\right)$ is vertex-pancyclic, we have only to show that an element from $\mathcal{S}_{0}$ can belong to a cycle of even length $\ell \in\{4,6, \ldots$, $\left.2 n^{2}-n-1\right\}$. To show that this is possible, we can modify $\mathcal{P}_{k, e, i}$, for even $e \geq 4$, to be

$$
\begin{aligned}
& \mathcal{P}_{k, e, i}^{\prime} \\
& :=S_{k, i}^{\prime} \rightarrow S_{0, i} \rightarrow S_{0, i+1} \rightarrow S_{k+1, i} \rightarrow \cdots \rightarrow S_{k+\frac{e}{2}-2, i} \rightarrow S_{k+\frac{e}{2}-2, i}^{\prime} \rightarrow S_{k, i}
\end{aligned}
$$




\section{ZERO-DIVISOR GRAPHS OF $2 \times 2$ MATRIX RINGS OVER $\mathbb{Z}_{n}$}

We can simply change between $\mathcal{P}_{k, e, i}$ and $\mathcal{P}_{k, e, i}^{\prime}$ as necessary. It follows that $\Gamma\left(T_{2}(n)\right)$ is vertex-pancyclic.

Now suppose $n$ is composite. Let $p_{1}$ be the smallest prime factor of $n$, and let

$$
Q=\left\{\left(\begin{array}{cc}
a & 0 \\
0 & p_{1}
\end{array}\right): \operatorname{gcd}(a, n)=1\right\} .
$$

If $A \in Q$ and $B \in Z_{2}(n)$, then $A B=\mathbf{0}$ if and only if $B=\left(\begin{array}{cc}0 & 0 \\ 0 & \frac{k n}{p_{1}}\end{array}\right)$ for some $1 \leq k \leq p_{1}-1$. But since $|Q|=\phi(n)>p_{1}-1, \Gamma\left(T_{2}(n)\right)$ contains no cycle of length $\left|Z_{2}(n)\right|$. Thus $\Gamma\left(T_{2}(n)\right)$ is not pancyclic (and hence not vertex-pancyclic).

Corollary 3.18. $\Gamma\left(T_{2}(n)\right)$ is Hamiltonian if and only if $n$ is prime.

Proof. If $n=2$, the result is clear from the $\mathcal{S}, S^{\prime}$ diagram, and if $n$ is an odd prime, then it follows from Theorem 3.17 that $\Gamma\left(T_{2}(n)\right)$ is Hamiltonian. If $n$ is not prime, then $\Gamma\left(T_{2}(n)\right)$ has no cycle of length $\left|Z_{2}(n)\right|$ by the proof of Theorem 3.17, so $\Gamma\left(T_{2}(n)\right)$ is not Hamiltonian.

Definition 3.19. The clique number of a graph $G$, which we denote by $\omega(G)$, is the greatest integer $r \geq 1$ such that $K^{r} \subseteq G$, where $K^{r}$ is the complete graph on $r$ vertices.

Our final theorem shall give an explicit formula for the clique number of $\Gamma\left(T_{2}(n)\right)$. However, we must first establish two lemmas.

Lemma 3.20. Let $n=p_{1}^{e_{1}} p_{2}^{e_{2}} \cdots p_{m}^{e_{m}}$ be the prime factorization of $n$, let $S=\left\{s \in \mathbb{Z}_{n}: s^{2} \not \equiv 0(\bmod n)\right\}$, and let $C$ be a subset of $\mathbb{Z}_{n}$ whose elements form a complete subgraph of $\Gamma\left(\mathbb{Z}_{n}\right)$. Then $|S \cap C| \leq m$.

Proof. Let $x, y \in C \bigcap S$, and write $\operatorname{gcd}(x, n)=p_{1}^{a_{1}} \cdots p_{m}^{a_{m}}, \operatorname{gcd}(y, n)=$ $p_{1}^{b_{1}} \cdots p_{m}^{b_{m}}$. Since $x^{2} \not \equiv 0(\bmod n)$, there is some $i \in\{1, \ldots, m\}$ such that $a_{i}<e_{i} / 2$. Similarly, there is some $j \in\{1, \ldots, m\}$ such that $b_{j}<e_{j} / 2$. But since $x y \equiv 0(\bmod n)$, we have $i \neq j$, and it follows that $|S \cap C| \leq m$.

Lemma 3.21. Let $n \geq 2$, let $\mathcal{C}$ be a complete subgraph of $\Gamma\left(T_{2}(n)\right)$, and define $\mathcal{G}=\left\{\left\{a_{i j}\right\} \in \mathcal{C}: a_{11}=a_{22}=0\right\}$. If $\operatorname{gcd}\left(a_{12}, n\right)=1$ for any $\left\{a_{i j}\right\} \in \mathcal{G}$, then $\mathcal{C}=\mathcal{G}$.

Proof. Suppose $A=\left\{a_{i j}\right\} \in \mathcal{G}$ with $\operatorname{gcd}\left(a_{12}, n\right)=1$, and suppose $B=$ $\left\{b_{i j}\right\} \in \mathcal{C}$ such that $B \neq A$. Since $A B=\mathbf{0}$, we have $a_{12} b_{22}=0$, which implies $b_{22}=0$. Similarly, $B A=\mathbf{0}$ implies $b_{11}=0$. Thus, $B \in \mathcal{G}$, and it follows that $\mathcal{C}=\mathcal{G}$.

Recall that $K$ is a complete subgraph of a directed graph $G$ if $A \rightarrow B$ and $B \rightarrow A$ for every pair of distinct vertices $A, B$ of $K$.

MISSOURI J. OF MATH. SCI., FALL 2014 


\section{T. FENSTERMACHER AND E. GEGNER}

Theorem 3.22. Let $n=p_{1}^{e_{1}} p_{2}^{e_{2}} \cdots p_{m}^{e_{m}}$ be the prime factorization of $n$. If $n=2$, then $\omega\left(\Gamma\left(T_{2}(n)\right)\right)=2$; otherwise,

$$
\omega\left(\Gamma\left(T_{2}(n)\right)\right)=\xi n-1,
$$

where $\xi=p_{1}^{\left\lfloor\frac{e_{1}}{2}\right\rfloor} p_{2}^{\left\lfloor\frac{e_{2}}{2}\right\rfloor} \cdots p_{m}^{\left\lfloor\frac{e_{m}}{2}\right\rfloor}$.

Proof. The $\mathcal{S}, \mathcal{S}^{\prime}$ diagram verifies Theorem 3.22 when $n=2$. Thus, suppose $n \geq 3$, and let $H=\left\{h \in \mathbb{Z}_{n}: h^{2} \equiv 0(\bmod n)\right\}$. Note that $|H|=\xi$. We now create a complete subgraph in $\Gamma\left(T_{2}(n)\right)$; let

$$
\Omega=\left\{\left(\begin{array}{cc}
a & b \\
0 & c
\end{array}\right) \neq \mathbf{0}: a, c \in H, b=k \xi \text { for } 0 \leq k<\frac{n}{\xi}\right\} .
$$

Note that the elements of $\Omega$ form a complete subgraph of $\Gamma\left(T_{2}(n)\right)$, and $|\Omega|=\xi n-1$. We claim that the elements of $\Omega$ form a complete subgraph in $\Gamma\left(T_{2}(n)\right)$ of maximum order.

Suppose that $\mathcal{C}$ is a complete subgraph of $\Gamma\left(T_{2}(n)\right)$. Define the sets

$$
\begin{aligned}
H_{1} & =\left\{a_{11} \in H:\left\{a_{i j}\right\} \in \mathcal{C}\right\} \\
H_{2} & =\left\{a_{22} \in H:\left\{a_{i j}\right\} \in \mathcal{C}\right\} \\
S_{1} & =\left\{\operatorname{gcd}\left(a_{11}, n\right):\left\{a_{i j}\right\} \in \mathcal{C}, a_{11} \notin H\right\} \\
S_{2} & =\left\{\operatorname{gcd}\left(a_{22}, n\right):\left\{a_{i j}\right\} \in \mathcal{C}, a_{22} \notin H\right\} .
\end{aligned}
$$

Note that $\left|S_{1}\right|+\left|S_{2}\right|$ is an upper bound for the number of matrices in $\mathcal{C}$ with at most one diagonal entry in $H$. For if $B=\left\{b_{i j}\right\}, C=\left\{c_{i j}\right\}$ and $\operatorname{gcd}\left(b_{k k}, n\right)=\operatorname{gcd}\left(c_{k k}, n\right) \notin H$ for some $k \in\{1,2\}$, then $B C \neq \mathbf{0}$, since $\left(b_{k k}\right)\left(c_{k k}\right) \not \equiv 0(\bmod n)$. Since $n \geq 3$, Lemma 3.20 implies $\left|S_{1}\right|+\left|S_{2}\right| \leq$ $2 m \leq n-1$.

Let $z=n / \xi$, and for each $k \in\{1,2\}$, let $\mathcal{P}_{k}=\left\{\left\{a_{i j}\right\} \in \mathcal{C}: a_{k k} \in H_{k}\right\}$, $h_{k}=\operatorname{gcd}\left(v: v \in H_{k} \bigcup\{n\}\right)$. Notice that $h_{k} \geq z$ for $k \in\{1,2\}$, and that $\left|H_{k}\right| \leq n / h_{k}$ for $k \in\{1,2\}$, where equality implies $0 \in H_{k}$. For all $x, y \in \mathbb{Z}_{\xi}$, define

$$
\begin{aligned}
& \mathcal{F}_{x, y}=\left\{\left\{a_{i j}\right\} \in \mathcal{C}: a_{11}=x z \text { and } a_{22}=y z\right\} \\
& \mathcal{G}_{x, y}=\left\{a_{12}: a_{11}=x z \text { and } a_{22}=y z\right\} .
\end{aligned}
$$

If $\mathcal{C}=\mathcal{F}_{0,0}$, then $|\mathcal{C}| \leq n-1 \leq \xi n-1$. So assume $\mathcal{C} \neq \mathcal{F}_{0,0}$. Suppose $x_{1}, x_{2} \in \mathbb{Z}_{\xi}$. We claim that $\left|\mathcal{F}_{x_{1}, x_{2}}\right| \leq \min \left\{h_{1}-1, h_{2}-1\right\}$ if $x_{1}=x_{2}=0$, and $\left|\mathcal{F}_{x_{1}, x_{2}}\right| \leq \min \left\{h_{1}, h_{2}\right\}$ otherwise. We first consider the case $\left|\mathcal{F}_{x_{1}, x_{2}}\right| \leq 2$. Since $n \geq 3$, we must have $h_{1}, h_{2} \geq 2$, so if $x_{1}, x_{2}$ are not both zero, we are done. Suppose $x_{1}=x_{2}=0$. If $\min \left\{h_{1}, h_{2}\right\}>2$ we are done, so suppose $\min \left\{h_{1}, h_{2}\right\}=2$. Then $n=4$, and since $\mathcal{C} \neq \mathcal{F}_{0,0}$, Lemma 3.21 implies that $1,3 \notin \mathcal{G}_{x_{1}, x_{2}}$, so $\left|\mathcal{F}_{x_{1}, x_{2}}\right|=\left|\mathcal{G}_{x_{1}, x_{2}}\right| \leq 1$. This proves the claim for $\left|\mathcal{F}_{x_{1}, x_{2}}\right| \leq 2$. 
ZERO-DIVISOR GRAPHS OF $2 \times 2$ MATRIX RINGS OVER $\mathbb{Z}_{n}$

Now suppose $\left|\mathcal{F}_{x_{1}, x_{2}}\right| \geq 3$. Let $B=\left\{b_{i j}\right\}, C=\left\{c_{i j}\right\} \in \mathcal{F}_{x_{1}, x_{2}}$. To prove $\left|\mathcal{F}_{x_{1}, x_{2}}\right| \leq h_{1}$, suppose $D=\left\{d_{i j}\right\} \in \mathcal{P}_{1}$ such that $D \neq B, D \neq C$. Then $D=\left(\begin{array}{cc}r_{D} h_{1} & d_{12} \\ 0 & d_{22}\end{array}\right)$ for some $r_{D} \in \mathbb{Z}_{\xi}$. Since $D B=D C=\mathbf{0}$, it follows that

$$
r_{D} h_{1} b_{12}+d_{12} x_{2} z \equiv r_{D} h_{1} c_{12}+d_{12} x_{2} z \equiv 0 \quad(\bmod n)
$$

which implies

$$
r_{D} b_{12} \equiv r_{D} c_{12} \quad\left(\bmod n / h_{1}\right)
$$

Let $T=\left\{c_{12}, c_{12}+n / h_{1}, c_{12}+2 n / h_{1}, \ldots, c_{12}+n-n / h_{1}\right\}$, where the values in $T$ are taken modulo $n$. Since equation (3.7) must hold for all $D \in \mathcal{P}_{1}$, and since $\left|\mathcal{F}_{x_{1}, x_{2}}\right| \geq 3$, it follows that $r b_{12} \equiv r c_{12}\left(\bmod n / h_{1}\right)$ holds for all $r$ such that $r h_{1} \in H_{1}$. But $\operatorname{gcd}\left(r: r h_{1} \in H_{1}\right)=1$, so $b_{12} \in T$. Thus, $\mathcal{G}_{x_{1}, x_{2}} \subseteq T$ and $\left|\mathcal{F}_{x_{1}, x_{2}}\right|=\left|\mathcal{G}_{x_{1}, x_{2}}\right| \leq|T|=h_{1}$. Note that if $x_{1}=x_{2}=0$, then $0 \in T$. However, $0 \notin \mathcal{G}_{0,0}$, so $\left|\mathcal{F}_{0,0}\right| \leq h_{1}-1$.

To prove that $\left|\mathcal{F}_{x_{1}, x_{2}}\right| \leq h_{2}$ and $\left|\mathcal{F}_{0,0}\right| \leq h_{2}-1$, suppose $A \in \mathcal{P}_{2}$ such that $A \neq B, A \neq C$. Then $A=\left(\begin{array}{cc}a_{11} & a_{12} \\ 0 & r_{A} h_{2}\end{array}\right)$ for some $r_{A} \in \mathbb{Z}_{\xi}$. Since $B A=C A=\mathbf{0}$, it follows that

$$
a_{12} x_{1} z+b_{12} r_{A} h_{2} \equiv a_{12} x_{1} z+c_{12} r_{A} h_{2} \equiv 0 \quad(\bmod n)
$$

which implies

$$
r_{A} b_{12} \equiv r_{A} c_{12} \quad\left(\bmod n / h_{2}\right)
$$

From here a similar argument as that used in the previous paragraph shows that $\left|\mathcal{F}_{x_{1}, x_{2}}\right| \leq h_{2}$ and $\left|\mathcal{F}_{0,0}\right| \leq h_{2}-1$.

Thus we have

$$
\begin{aligned}
|\mathcal{C}| & \leq \sum_{x \in H_{1}} \sum_{y \in H_{2}}\left|\mathcal{F}_{x, y}\right|+\left|S_{1}\right|+\left|S_{2}\right| \\
& \leq\left(\frac{n^{2}}{h_{1} h_{2}}-1\right) \min \left\{h_{1}, h_{2}\right\}+\min \left\{h_{1}-1, h_{2}-1\right\}+\left|S_{1}\right|+\left|S_{2}\right| .
\end{aligned}
$$

Suppose $\max \left\{h_{1}, h_{2}\right\}=z$, so that $\left|S_{1}\right|=\left|S_{2}\right|=0$. Then (3.10) becomes

$|\mathcal{C}| \leq\left(\frac{n^{2}}{h_{1} h_{2}}-1\right) \min \left\{h_{1}, h_{2}\right\}+\min \left\{h_{1}-1, h_{2}-1\right\}=\frac{n^{2}}{z}-z+z-1=\xi n-1$

On the other hand, if $\max \left\{h_{1}, h_{2}\right\}>z$, then $\xi=\frac{n}{z} \geq \frac{\max \left\{h_{1}, h_{2}\right\}}{z} \geq 2$, so

$$
\frac{n}{\max \left\{h_{1}, h_{2}\right\}}+1 \leq \frac{\xi}{2}+1 \leq \xi .
$$

MISSOURI J. OF MATH. SCI., FALL 2014 


\section{T. FENSTERMACHER AND E. GEGNER}

Thus,

$$
\begin{aligned}
|\mathcal{C}| & \leq \sum_{x \in H_{1}} \sum_{y \in H_{2}}\left|\mathcal{F}_{x, y}\right|+\left|S_{1}\right|+\left|S_{2}\right| \\
& <\left(\frac{n}{h_{1}}\right)\left(\frac{n}{h_{2}}\right) \min \left\{h_{1}, h_{2}\right\}+n-1 \\
& =\frac{n^{2}}{\max \left\{h_{1}, h_{2}\right\}}+n-1 \leq \xi n-1 .
\end{aligned}
$$

Therefore, no complete subgraph of $\Gamma\left(T_{2}(n)\right)$ has size greater than $\xi n-1$. This completes the proof that $\omega\left(\Gamma\left(T_{2}(n)\right)\right)=\xi n-1$.

\section{Acknowledgments}

The authors would like to thank Professor Jeremy Case for his guidance throughout this project, his careful review of the manuscript, and his valuable recommendations for its improvement.

\section{REFERENCES}

[1] D. D. Anderson and M. Naseer, Beck's coloring of a commutative ring, Journal of Algebra, 159.2 (1993), 500-514.

[2] D. F. Anderson, M. C. Axtell, and J. A. Stickles, Zero-divisor graphs in commutative rings, Commutative Algebra, Springer, New York, 2011.

[3] D. F. Anderson and P. S. Livingston, The zero-divisor graph of a commutative ring, Journal of Algebra, 217.2 (1999), 434-447.

[4] M. Artin, Algebra, Prentice Hall, New Jersey, 1991.

[5] I. Beck, Coloring of commutative rings, Journal of Algebra, 116.1 (1988), 208-226.

[6] M. Behboodi and R. Beyranvand, Strong zero-divisor graphs of non-commutative rings, International Journal of Algebra, 2.1 (2008), 25-44.

[7] B. Bollabás, Graph Theory, An Introductory Course, Springer-Verlag, New York, 1979.

[8] R. Grimaldi, Discrete and Combinatorial Mathematics: An Applied Introduction, Addison-Wesley, Longman, Mass., 1998.

[9] I. N. Herstein, Topics in Algebra, Blaisdell Pub., New York, 1964.

[10] T. Y. Lam, A First Course in Noncommutative Rings, Springer-Verlag, New York, 1991.

[11] I. Niven, H. Montgomery, and H. Zuckerman, An Introduction to the Theory of Numbers, Wiley, New York, 1991.

[12] S. Redmond, The zero-divisor graph of a non-commutative ring, Trends in Commutative Rings Research, Nova Science, New York, 2004.

[13] S. Redmond, On zero-divisor graphs of small finite commutative rings, Discrete Mathematics, 307.21 (2007), 1155-1166.

MSC2010: 05C40, 05C45 05C69, 16S50 
ZERO-DIVISOR GRAPHS OF $2 \times 2$ MATRIX RINGS OVER $\mathbb{Z}_{n}$

Key words and phrases: non-commutative ring, zero-divisor graph, clique number, edge-connectivity, Hamiltonian, upper-triangular matrices

TAYlor University, Upland, Indiana, 46989

E-mail address: todd_fenstermacher@taylor.edu

TAylor University, Upland, Indiana, 46989

E-mail address: ethan_gegner@taylor.edu 